\title{
Effect of Freezing Time and Shaking Speed on the Performance of Progressive Freeze Concentration via Vertical Finned Crystallizer
}

\author{
N. A. Amran ${ }^{1,2}$, S. Samsuri ${ }^{1,2}$ and M. Jusoh ${ }^{3 *}$ \\ ${ }^{1}$ Department of Chemical Engineering, Universiti Teknologi PETRONAS, \\ 32610 Seri Iskandar, Perak, Malaysia \\ ${ }^{2}$ Centre for Biofuel and Biochemical Research, Universiti Teknologi PETRONAS, \\ 32610 Seri Iskandar, Perak, Malaysia \\ ${ }^{3}$ Department of Chemical and Energy Engineering, Universiti Teknologi Malaysia, \\ 81310 UTM Skudai, Johor, Malaysia \\ *Email: mazura@cheme.utm.my \\ Phone: +6075535535; Fax: +6075588166
}

\begin{abstract}
Performance of a newly developed freeze concentrator named Vertical Finned Crystallizer (VFC) in concentrated glucose solution was evaluated. The VFC was designed as an attempt to provide an efficient Progressive Freeze Concentration (PFC) system by providing larger heat transfer area for crystallization. Glucose solution at an initial concentration of $11^{\circ}$ Brix was concentrated through the new PFC system. The performance of the crystallizer was analyzed in parallel with the effect of freezing time and shaking speed to the system efficiency, represented by effective partition constant $(K)$ and solute recovery $(Y)$. The results show that the efficiency of the system has significantly improved portrayed by the lowest $K$ value obtained of 0.383 and the highest $Y$ value obtained of $0.96 \mathrm{~g}$ glucose/g initial glucose at intermediate circulation time and shaking speed. Thus, the VFC is evidently proven to have a high potential to be integrated in a PFC process as an efficient concentration system. The results obtained offer some guidelines on how to produce an efficient PFC system through parameter setting.
\end{abstract}

Keywords: Freeze concentration; cryoconcentration; progressive freeze concentration; vertical finned crystallizer; effective partition constant; solute recovery.

\section{INTRODUCTION}

Freeze Concentration (FC) is a process used to concentrate a solution by freezing water into pure ice and eliminating solutes or impurities from the formed ice simultaneously [1-7]. The greatest attraction of FC is it could preserve the thermally sensitive components in the concentrate due to the low process temperatures involved, as well as low energy requirement $(0.33 \mathrm{~kJ} / \mathrm{g}$ water $)$ as compared to the conventional concentration method by evaporation $[1,8]$. Based on this advantage, this process has now become favourable concentration method especially in liquid food processing industries as the nutritional quality of freeze concentrated products is higher than those concentrated by evaporation [9-11].

According to Flesland [12] and Miyawaki [13], there are two basic types of freeze concentration, which are suspension freeze concentration (SFC) and progressive freeze concentration (PFC). In SFC, small size ice crystals are produced in the 
suspension of mother solution while PFC continuously produces ice crystal layer by layer on a cooled surface until it forms a single and large block of ice. Hence, separation of ice crystal from solution becomes much easier and cheaper in PFC since SFC requires many unit operations especially in separation section which involves washing and filtration [4, 13-15]. As PFC offers a simpler separation step, it has been suggested in recent studies to associate the future application of FC more with the progresses in the configuration of PFC system than SFC system [3, 16].

Although PFC is reported to be a better way to concentrate solutions, its productivity has been found to be lower than SFC. This is mainly due to the nature of the formation of ice layer which is normally very slow. The slow ice layer formation in PFC limits the commercialization of the process. Although PFC could offer a cheaper separation process, it would not be favored by the industry if the process would not yield a good productivity and efficiency. As a result, many studies have been and are being made to find the way to improve the efficiency and productivity of the PFC method. However, less number of studies on proposing or creating new PFC systems and crystallizers has been reported [9, 17-20]. In PFC, the most important and crucial part to ensure the productivity and separation efficiency is the crystallizer. Crystallizer is the apparatus in which the crystallization process takes place.

The objective of this study is to propose a new PFC crystallizer, namely vertical finned crystallizer (VFC), as an attempt to provide an efficient PFC system by providing larger heat transfer area for crystallization through the subjected fins. This paper discusses the performance evaluation of the VFC through the changes in effective partition constant $(K)$ and solute recovery $(Y)$ at different circulation flowrate and shaking speed.

\section{MATERIALS AND METHODS}

\section{Design of Vertical Finned Crystallizer (VFC)}

Generally, the VFC is divided into five parts which are body, fin, stand, lid and cooling jacket. Figure 1 shows the general schematic of the vertical finned crystallizer. During the process, crystallization took place inside the body of the crystallizer consisting the subjected fins, while cooling jacket provided place for circulated coolant around the crystallizer body. The whole system of the new crystallizer (VFC) was generally built in cylindrical shape and made of stainless steel. The full volume of the VFC is approximately $1.15 \mathrm{~L}$, with wall thickness and diameter of $1 \mathrm{~mm}$ and $8 \mathrm{~cm}$, respectively. Four vertical rectangular fins with $30 \mathrm{~cm}$ height, $2 \mathrm{~cm}$ length and $1.5 \mathrm{~cm}$ width were introduced onto the inner wall of the new crystallizer (VFC). The subjection of these fins has increased the heat transfer area by $63.7 \%$.

\section{Experimental Setup}

The actual experimental setup of the PFC process is illustrated in Figure 2. The setup comprises of the new crystallizer (VFC), refrigerated waterbath (Scientz, China), refractometer (MA871, Romania, Europe), feed tank, peristaltic pump (Cole-Palmer, Canada), linear shaker, silicone tubes (Masterflex, USA), temperature data acquisition tool, Picolog (Picolog USB TC-08, United Kingdom) and temperature display. The shaker was custom made to fit the size and weight of the crystallizer. The reading of electrical resistance (ohm) of the shaker was used to controlled the shaking speed. 
During the experimental run, the VFC was placed on a shaker at which the VFC was shaken at certain designated shaking speed. At the same time, the crystallizer was connected via two silicone tubes to a peristaltic pump and a feed tank, where the pump circulates the solution from the feed tank into the crystallizer at the desired flowrate, for a period of time. In order to provide cooling energy to the system, a refrigerated waterbath was used where it was connected via another two silicone tubes to the cooling jacket of the VFC. The coolant was placed in the waterbath and cooled to the desired temperature before being circulated within the cooling jacket. On the other hand, a temperature data logger (Picolog) was used to display the temperature recorded by six thermocouples installed on the VFC through a connected computer.

\section{Materials}

Glucose solutions at a concentration of $11^{\circ}$ Brix were used as the target solution to be concentrated. $11^{\circ}$ Brix was chosen based on its suitability to represent various industrial solutions [1, 21]. Initially, analytical grade glucose powder $(\mathrm{D}(+)$-Glucose) was purchased from IChem Solution Sdn. Bhd. The solution was prepared by mixing $220 \mathrm{~g}$ of analytical grade glucose powder with $1780 \mathrm{ml}$ of distilled water. As reported from previous research $[4,13]$, glucose solution has been widely or commonly used in studies which evaluated the performance of freeze concentration process. On the other hand, $50 \%(\mathrm{v} / \mathrm{v})$ of ethylene glycol-water solution was used as a coolant to supply cooling energy to the system.

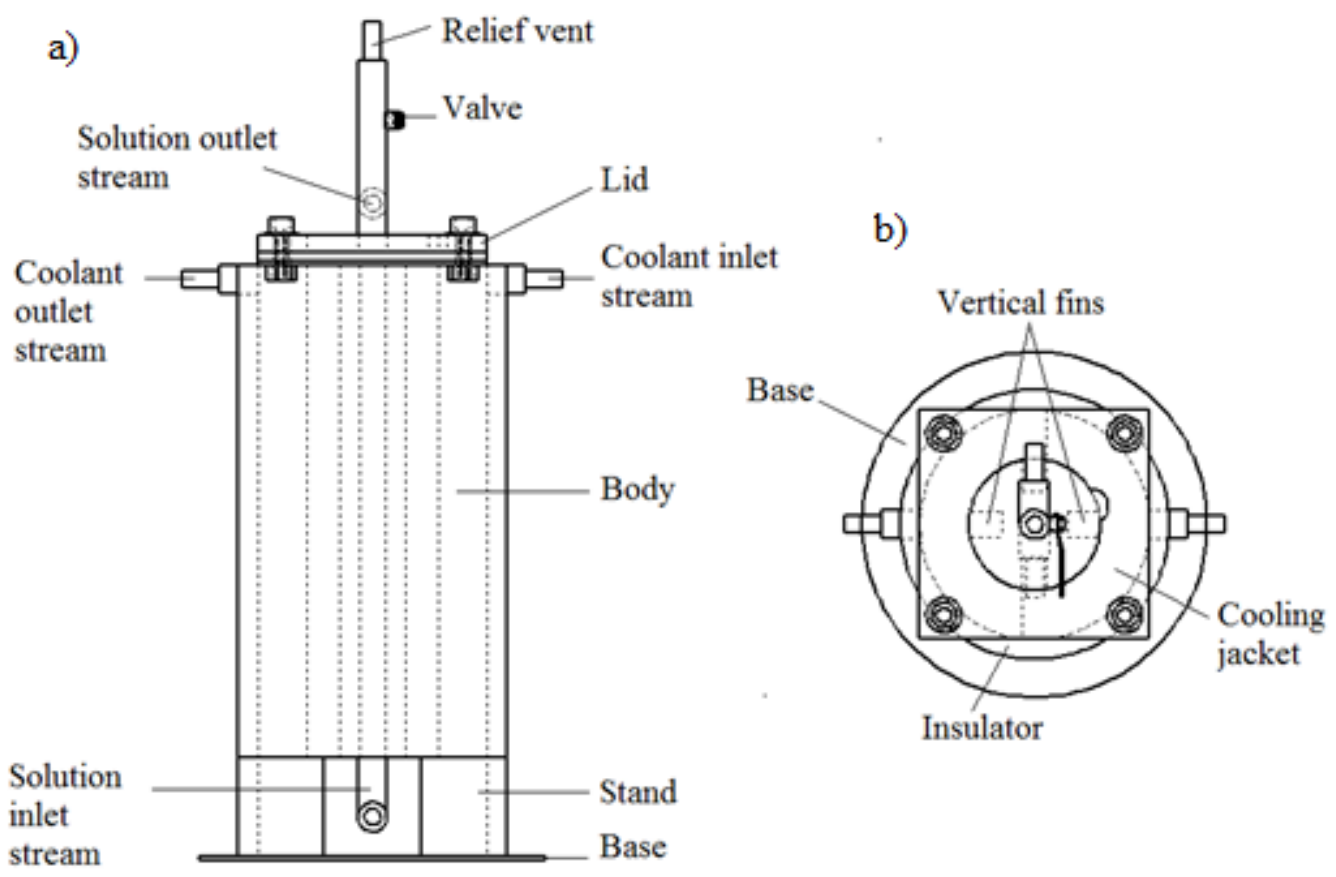

Figure 1. (a) Top and (b) side view of VFC. 


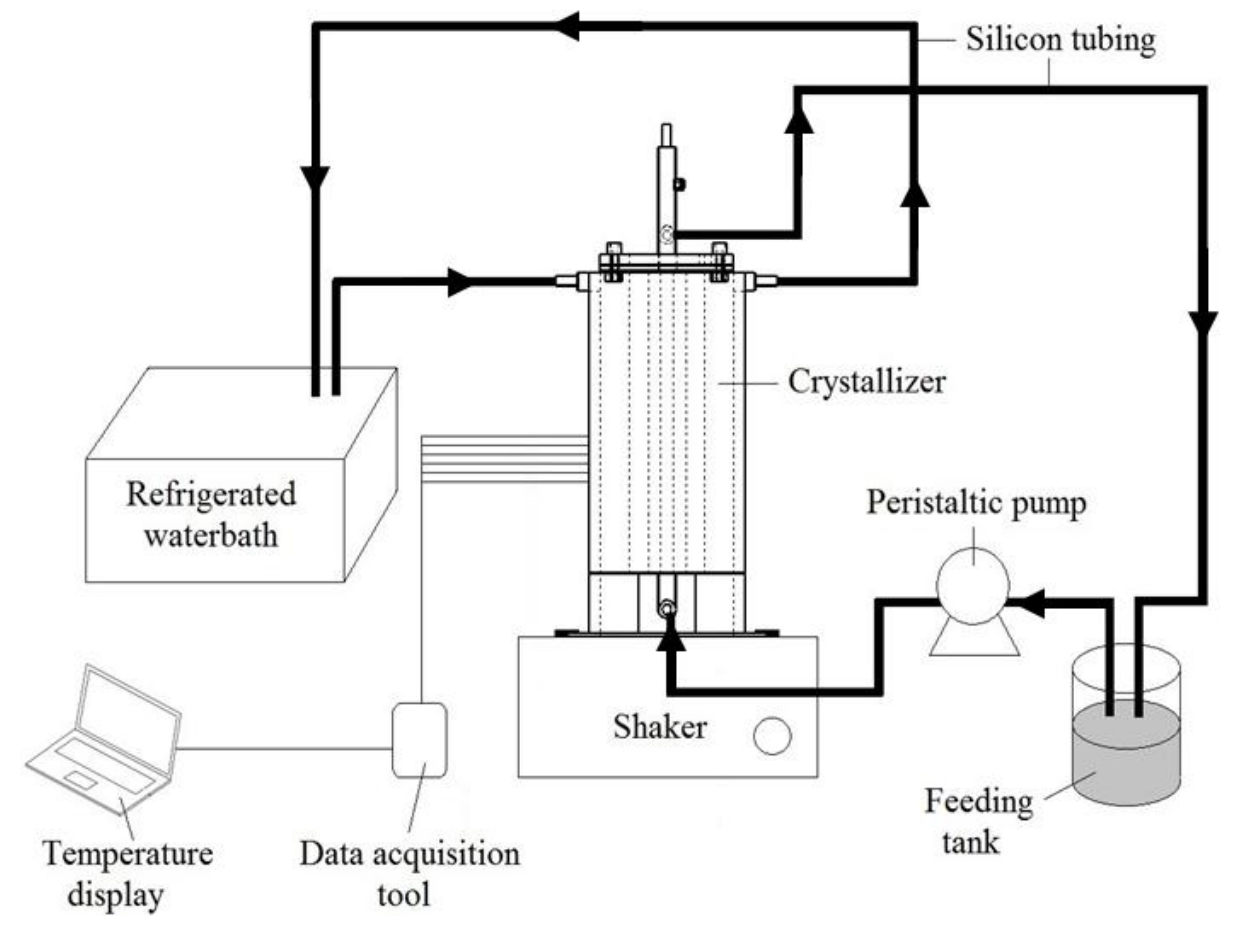

Figure 2. Experimental setup for the PFC system.

\section{Experimental Procedure}

Glucose solution was prepared at the desired concentration (Brix). Prior to be fed into the crystallizer for concentration purpose, the temperature of the glucose solution was kept near the freezing point of water. On the other hand, waterbath containing the coolant was switched on to reach coolant temperature of $-10^{\circ} \mathrm{C}$. As the coolant temperature reached the desired reading, the glucose solution was fed into the crystallizer from the feed tank using a peristaltic pump at $3400 \mathrm{~mL} / \mathrm{min}$ circulation flowrate. When the crystallizer and the silicone tube were filled with the solution, the feeding process from the feed tank was stopped by closing a valve located at the feed tank. At this time, the shaker was turned on at the desired shaking speed and the glucose solution was left in circulation within the crystallizer and the silicone tube for crystallization to occur.

After certain period, the circulation and shaking were stopped and the concentrated glucose solution in the crystallizer was collected from the crystallizer and silicone tube. For analysis purpose, volume of the ice layer formed and the concentrate were measured before collecting the samples. Then, the collected sample of ice and concentrate were analyzed for their glucose concentration using a refractometer. The experimental procedure was carried out in duplicates to provide an accurate result of experiment.

\section{Calculation of Effective Partition Constant $(K)$}

The efficiency of a PFC process is normally measured by an effective partition constant of a solute, $K$, which acts as an index for separation effectiveness. The value of $K$ changes between 0 (complete FC) and 1 (no FC) [4, 14, 22]. Lower value of $K$ indicates 
higher separation efficiency of the PFC process since concentration of solute in the ice formed is lower. In this study, the $K$ value was determined by using Eq. (1).

$K=1-\left[\log \left(C_{o} / C_{L}\right) / \log \left(V_{L} / V_{o}\right)\right]$

where $V_{o}$ is the initial volume of solution in $\mathrm{mL}$,

$V_{L}$ is the final volume of solution in $\mathrm{mL}$,

$C_{o}$ is the initial solute concentration of solution in ${ }^{\circ} \mathrm{Brix}$,

$C_{L}$ is the final solute concentration of solution in ${ }^{\circ} \mathrm{Brix}$.

\section{Calculation of Solute Recovery (Y)}

As the freezing process progresses in the crystallizer, solute concentration increases in the liquid portion [5, 23-25]. At the same time, an amount of the solute may also be entrapped in the ice phase, depending on the process conditions. Hence, the effectiveness of the process can also be defined by the solute recovery $(Y)$ which represents the amount of solute recovered in the concentrated liquid, from the original solution. According to Moreno et al. [26], solute recovery (Y) can be calculated by using Eq. (2):

$Y=M_{s, L} / M_{s, o}$

where $M_{s, L}$ is the mass of solute in the concentrated liquid in gm,

$M_{s, o}$ is the mass of solute in the initial solution in $\mathrm{gm}$.

\section{RESULTS AND DISCUSSION}

\section{Ice Crystal Formation}

During the freezing process, ice crystal layer has been successfully formed on the inner wall of the new crystallizer, thus proving the effectiveness of the design aspects of the Vertical Finned Crystallizer (VFC) for freeze concentration. Figure 3 shows the closeup view inside the VFC before (a) and after (b) the formation of ice crystal layer.

Through the experimental works, the volume of ice produced $\left(V_{S}\right)$ was measured together with the volume of concentrate collected $\left(V_{L}\right)$, as well as concentration of glucose in both concentrate $\left(C_{L}\right)$ and ice $\left(C_{S}\right)$ at the end of experiment. These values were later being used in the calculation of effective partition constant $(K)$ and solute recovery $(Y)$. Table 1 shows the calculated values of effective partition constant $(K)$ and solute recovery $(Y)$ at different circulation time and shaking speed of shaker. 


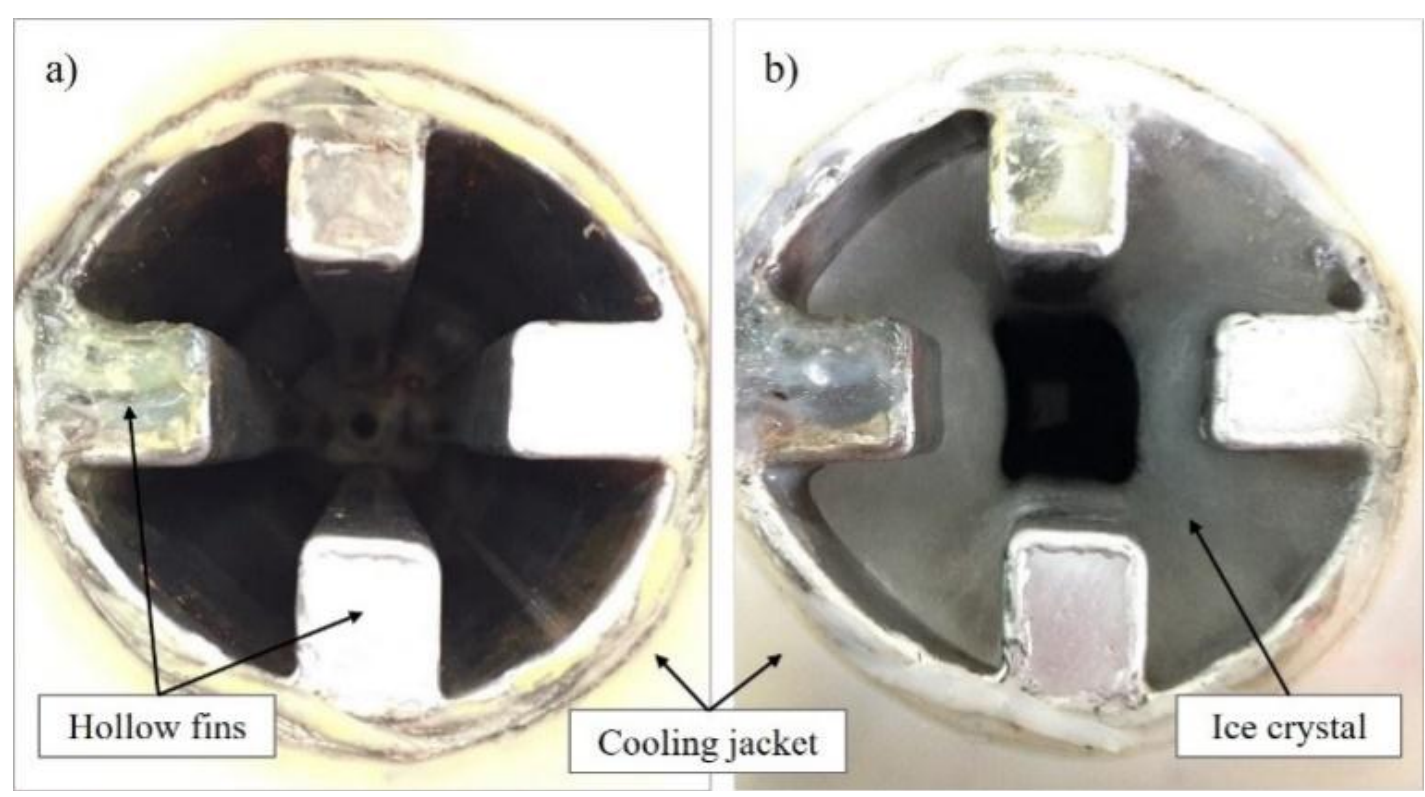

Figure 3. A close-up view of the VFC (a) before and; (b) after the formation of ice.

Table 1. Changes in $K$ and $Y$ value at different coolant temperature and circulation flow rate.

\begin{tabular}{cccccc}
\hline $\begin{array}{c}\text { Circulation } \\
\text { time (min) }\end{array}$ & $K$ & $Y$ & $\begin{array}{c}\text { Shaking } \\
\text { speed (ohm) }\end{array}$ & $K$ & $Y$ \\
\hline 40 & 0.568 & 0.945 & 30 & 0.581 & 0.890 \\
50 & 0.383 & 0.960 & 40 & 0.383 & 0.960 \\
60 & 0.476 & 0.948 & 50 & 0.395 & 0.948 \\
70 & 0.500 & 0.943 & 60 & 0.494 & 0.937 \\
80 & 0.517 & 0.938 & 70 & 0.525 & 0.907 \\
\hline
\end{tabular}

\section{Effect of Circulation Time}

Generally, longer circulation time could provide higher concentration efficiency to the PFC process $[3,11,27]$. As the freezing process started, the degree of crystallinity of the formed ice layer is still low with the presence of dendrites structure. By providing longer circulation time, the ice layer grows thicker, leaving the unfrozen solution in a state approaching the saturation level [28-31]. Figure 4 shows the trends of effective partition constant $(K)$ and solute recovery $(Y)$ at different circulation time.

From Figure 4, it can be observed that the $K$ value decreases as the circulation time increases from 40 to 50 minutes. In this analysis, a 50 minutes' circulation is sufficient to give the highest efficiency of the process, portrayed by the lowest $K$ value (0.383) and the highest $Y$ value (0.96) obtained. At this condition, the value of $Y$ describes that $96 \%$ of solute in the initial solution has been recovered in the concentrate. However, the trend was changed after 50 minutes of circulation where the $K$ value started to increase. The figure also shows that the solute recovery, $Y$ has an inverse relationship to the $K$ value. The value of $K$ obtained in this analysis is lower than the value obtained by Jusoh [32] which is 0.48 at maximum circulation time of 20 minutes. Although the lower value of $K$ was obtained at a higher circulation flowrate, 
the capability of VFC to yield an efficient separation process was proven through this value.

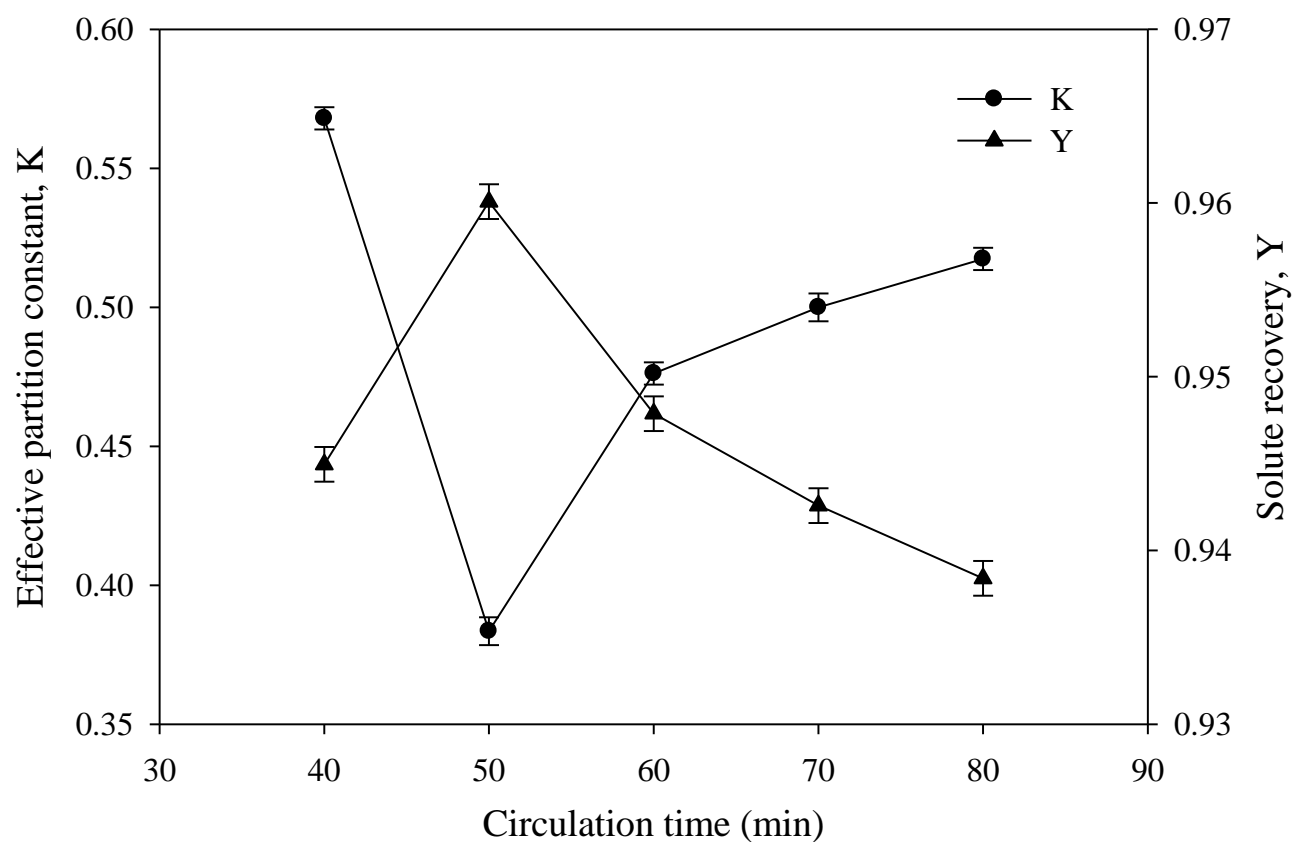

Figure 4. Changes in $K$ and $Y$ values at different circulation time

However, longer circulation time might shift the trend of solute concentration in the ice caused by the saturation of solute in the concentrate. This situation can be explained by the growth of ice layers which nearly occupied the space inside the crystallizer especially after 50 minutes of concentration process [16, 33]. In fact, this situation is supported by Chen et al. $[34,35]$ in their study which stated that higher solid fraction could give lower ice purity. Hence, high amount of solutes in the concentrate can easily be trapped into the ice layers if the concentration process continued, thus lowering the amount of solute recovered in the concentrate as well as process efficiency.

\section{Effect of Shaking Speed}

In this study, a shaker was used particularly to distribute and mix the solutes well in the target solution which later could enhance the production of highly concentrated concentrate as well as highly purified ice. Figure 5 shows the trends of effective partition constant $(K)$ and solute recovery $(Y)$ at different circulation time. The value of $K$ decreases as the shaking speed increases from 30 to $40 \mathrm{ohm}$, with $40 \mathrm{ohm}$ recording the lowest $K$ value portraying the highest efficiency of the system in this particular analysis. Similar trend was also obtained in a study by Iritani et al. [36]. However, the value of $K$ starts to increase as the shaking speed further increases up to $70 \mathrm{ohms}$. On the other hand, the observed trend of solute recovery $(Y)$ is opposite to the trend observed for $K$ value. 


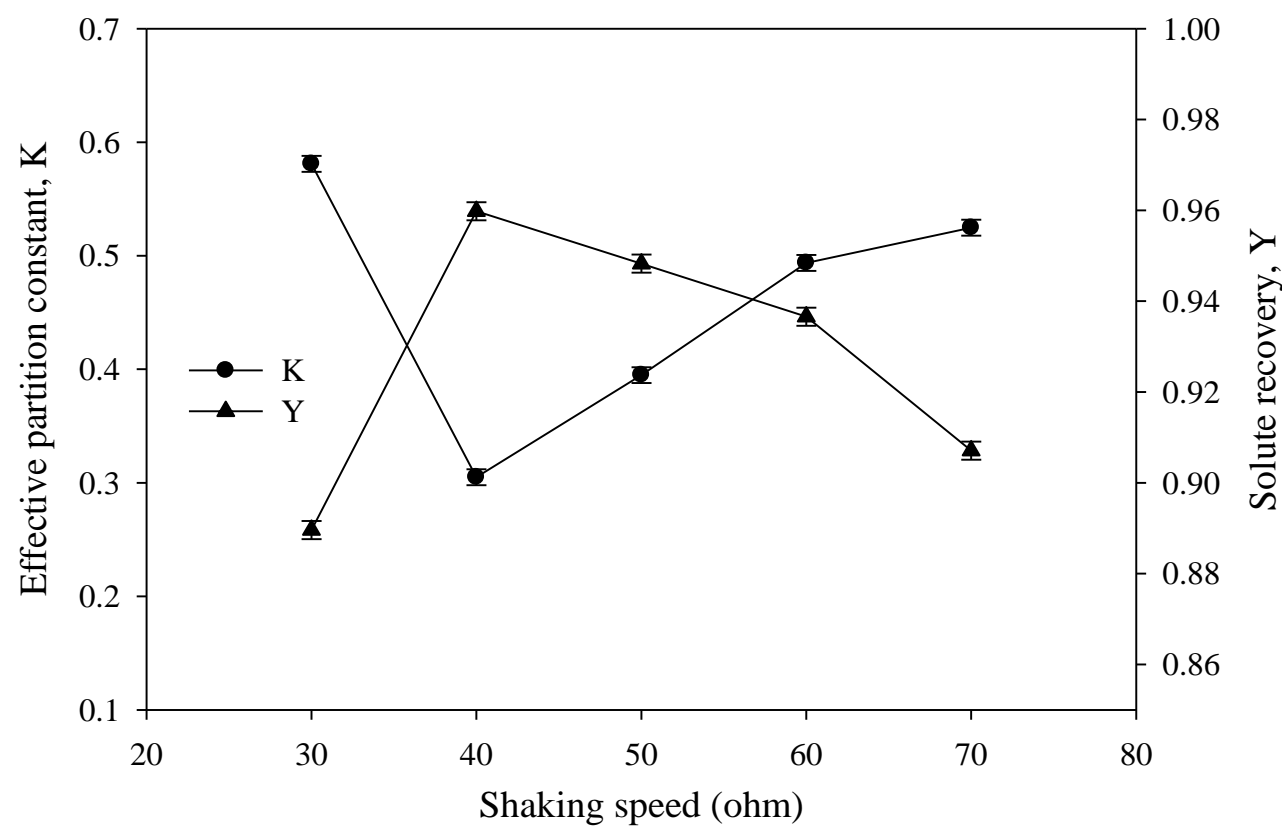

Figure 5. Changes in $K$ and $Y$ values at different shaking speed

Generally, a higher separation efficiency was produced at higher mixing rate $[2$, 37, 38]. This phenomenon is in line with the concentration polarization model developed by Miyawaki et al. [13, 39] which stated that higher mixing rate can reduce the extent of solute accumulation at the ice-liquid interface. Hence, the number of solute trapped into the ice could be reduced. This phenomenon explains clearly the decrease in $K$ value as well as the increase in $Y$ value as the shaking speed was increased from 30 to $40 \mathrm{ohm}$. When the amount of trapped solute in the ice was low at higher shaking speed, it also shows that the amount of solute recovered in the concentrate was high.

However, further increase of the shaking speed had caused a decrease in separation efficiency portrayed by higher value of $K$ and lower value of $Y$. This result can be explained by a reduction in the volume of ice produced when the shaking speed of shaker was increased. Although solute distribution in the solution could be enhanced by providing a shaker to the system, too vigorous shaking effect could interrupt the formation of ice on the wall of the crystallizer. This situation can be best explained by the fact that the front growth of the ice is in slushy form where it can be easily washed away or eroded from the rigid ice layer by the vigorous shaking $[36,40]$.

\section{CONCLUSION}

In PFC, it is important to understand the kinetics of the impurity or solute incorporation in the crystal formed during the crystallization, as well as to observe the relationship between process parameters and the impurity concentration in the ice crystal. Studies on theoretical and experimental analysis on the effect of parameters towards the system efficiency are important in improving the performance of the process itself. The lowest obtained value of effective partition constant $(K)$ and the highest obtained value of solute recovery $(Y)$ from this study have proven that the newly designed crystallizer, namely vertical finned crystallizer (VFC) is capable to concentrate glucose solution through progressive freeze concentration (PFC) process. The performance of the system which has been evaluated through behavior identification of effective partition constant $(K)$ and solute recovery $(Y)$ has shown that the VFC has high potential and is ready to 
compete with the previous existing PFC designs. The two determinant parameters $(K$ and $Y$ ) were also found to be dependent on the circulation time and shaking speed of shaker. As elaborated in the previous section, adequate circulation time (50 minutes) is needed to give the highest efficiency of the process. In addition, providing intermediate shaking speed $(40 \mathrm{ohm})$ is a good technique to distribute and mix the solutes well in the target solution which later could enhance the production of highly concentrated concentrate as well as highly purified ice. Through this study, the best effective partition constant $(K)$ and solute recovery $(Y)$ obtained are 0.383 and $0.96 \mathrm{~g}$ glucose/g initial glucose, respectively.

\section{ACKNOWLEDGEMENT}

The financial supports offered by Universiti Teknologi PETRONAS, Universiti Teknologi Malaysia and Ministry of Education Malaysia (MOE) are gratefully acknowledged.

\section{REFERENCES}

[1] Jusoh M. Development of a novel system for progressive freeze concentration process. Skudai: Universiti Teknologi Malaysia; 2010.

[2] Shapiro J. Freezing-out, a safe technique for concentration of dilute solutions. Science (New York, NY), 1961;133:2063-4.

[3] Amran NA, Jusoh M. Effect of Coolant Temperature and Circulation Flowrate on the Performance of a Vertical Finned Crystallizer. Procedia Engineering, 2016;148:1408-15.

[4] Miyawaki O, Liu L, Shirai Y, Sakashita S, Kagitani K. Tubular ice system for scale-up of progressive freeze-concentration. Journal of Food Engineering, 2005;69:107-13.

[5] Ab. Hamid FH, Rahim NA, Johari A, Ngadi N, Zakaria ZY, Jusoh M. Desalination of seawater through progressive freeze concentration using a coil crystallizer. Water Science and Technology: Water Supply, 2015;15:625-31.

[6] Buss DD. Milk goes arctic: Freeze-concentrated milk is first major alternative to thermally evaporated milk. Food Processing, 1993:62-4.

[7] Deshpande SS, Cheryan M, Sathe SK, Salunkhe DK, Luh BS. Freeze concentration of fruit juices. C R C Critical Reviews in Food Science and Nutrition, 1984;20:173-248.

[8] Shirai Y, Wakisaka M, Miyawaki O, Sakashita S. Conditions of Producing an Ice Layer with High Purity for Freeze Wastewater Treatment. Journal of Food Engineering, 1998;297-308.

[9] Aider M, de Halleux D. Cryoconcentration technology in the bio-food industry: Principles and applications. LWT - Food Science and Technology, 2009;42:67985.

[10] Jusoh M, Mohd Yunus R, Abu Hassan MA. Performance Investigation on a New Design for Progressive Freeze Concentration System. Journal of Applied Sciences, 2009;9:3171-5.

[11] Safiei NZ, Ngadi N, Johari A, Zakaria ZY, Jusoh M. Grape Juice Concentration by Progressive Freeze Concentrator Sequence System. Journal of Food Processing and Preservation, 2017;41:e12910-n/a. 
[12] Flesland OLA. Freeze concentration by layer crystallization. Drying Technology, 1995;13:1713-39.

[13] Miyawaki O. Progressive freeze-concentration: A new method for high-quality concentration of liquid food. Food Engineering Program, 2001;5:190-4.

[14] Liu L, Miyawaki O, Nakamura K. Progressive Freeze Concentration of Model Liquid Food. Food Science and Technology International, 1997;3:348-52.

[15] Rodriguez M, Luque S, Alvarez JR, Coca J. A comparative study of reverse osmosis and freeze concentration for the removal of valeric acid from wastewaters. Desalination, 2000;127:1-11.

[16] Amran NA. Development of a vertical finned crystallizer for progressive freeze concentration process. Skudai, Malaysia: Universiti Teknologi Malaysia; 2015.

[17] Nonthanum P, Tansakul A. Freeze concentration of lime juice Journal of Science and Technology, 2008;1:27-37.

[18] Bayindirli L, Özilgen M, Ungan S. Mathematical analysis of freeze concentration of apple juice. Journal of Food Engineering, 1993;19:95-107.

[19] Fujioka R, Wang LP, Dodbiba G, Fujita T. Application of progressive freezeconcentration for desalination. Desalination, 2013;319:33-7.

[20] Ramos FA, Delgado JL, Bautista E, Morales AL, Duque C. Changes in volatiles with the application of progressive freeze-concentration to Andes berry (Rubus glaucus Benth). Journal of Food Engineering, 2005;69:291-7.

[21] Viet Bui A, Nguyen b MH. Prediction of viscosity of glucose and calcium chloride solutions. Journal of Food Engineering, 2004;62:345-9.

[22] Liu L, Miyawaki O, Hayakawa K. progressive freeze concentration of tomato juice. Food Science and Technology International, 1999;5:108-12.

[23] Jusoh M, Mohd. Yunus R, Abu Hassan MA. Efficiency study on a new progressive freeze concentration system for freeze wastewater treatment. Jurnal Teknologi, 2008;49:261-70.

[24] Pradistsuwana C, Theprugsa P, Miyawaki O. Measurement of limiting partition coefficient in progressive freeze-concentration. Food Science and Technology Research, 2003;9:190-2.

[25] Halde R. Concentration of impurities by progressive freezing. Water Research, 1979;14:575-80.

[26] Moreno FL, Hernández E, Raventós M, Robles C, Ruiz Y. A process to concentrate coffee extract by the integration of falling film and block freezeconcentration. Journal of Food Engineering, 2014;128:88-95.

[27] Sanchez J, Ruiz Y, Raventos M, Auleda JM, Hernandez E. Progressive freeze concentration of orange juice in a pilot plant falling film. Innovative Food Science and Emerging Technologies, 2010;11:644-51.

[28] Chianese A, Santilli N. Modelling of the solid layer growth from melt crystallization - the integral formulation approach. chemical Engineering Science, 1998;53:107-11.

[29] Zambrano A, Ruiz Y, Hernández E, Raventós M, Moreno FL. Freeze desalination by the integration of falling film and block freeze-concentration techniques. Desalination, 2018;436:56-62.

[30] Osorio M, Moreno FL, Raventós M, Hernández E, Ruiz Y. Progressive stirred freeze-concentration of ethanol-water solutions. Journal of Food Engineering, 2018;224:71-9. 
[31] Yang Y, Lu Y, Guo J, Zhang X. Application of freeze concentration for fluoride removal from water solution. Journal of Water Process Engineering, 2017;19:260-6.

[32] Jusoh M, Mohd Yunus R. , A. AHM. Development of a New Crystallisation Chamber for a Progressive Freeze Concentration System. Recent Advance in Technology, 2009:587-600.

[33] Orellana-Palma P, Petzold G, Pierre L, Pensaben JM. Protection of polyphenols in blueberry juice by vacuum-assisted block freeze concentration. Food and Chemical Toxicology, 2017.

[34] Chen P, Chen XD, Free KW. Solute Inclusion in Ice Formed From Sucrose Solutions on a Sub-cooled Surface - An Experimental Study. Journal of Food Engineering, 1998;38:1-13.

[35] Chen YH, Cao E, Cui ZF. An Experimental Study of Freeze Concentration in Biological Media. Food and Bioproducts Processing, 2001;79:35-40.

[36] Iritani E, Katagiri N, Okada K, Cao D-Q, Kawasaki K. Improvement of concentration performance in shaking type of freeze concentration. Separation and Purification Technology, 2013;120:445-51.

[37] Md Zamani SH, Yahya N, Zakaria ZY, Jusoh M. Fractional freezing of ethanol and water mixture. Jurnal Teknologi, 2015;74:49-52.

[38] Hernandez E, Raventos M, Auleda JM, Ibarz A. Concentration of apple and pear juices in a multi-plate freeze concentrator. Innovative Food Science and Emerging Technologies, 2009;10:348-55.

[39] Miyawaki O, Liu L, Nakamura K. Effective Partition Constant of Solute between Ice and Liquid Phases in Progressive Freeze-Concentration. Journal of Food Science, 1998;63:756-8.

[40] Keshani S, Luqman Chuah A, Nourouzi MM, Russly AR, Jamilah B. Optimization of concentration process on pomelo fruit juice using response surface methodology (RSM). International Food Research Journal, 2010;17:73342. 